\section{RMD Open}

Rheumatic \&

Musculoskeletal Diseases

To cite: Hedström AK, Hössjer 0, Klareskog L, et al. Interplay between alcohol, smoking and HLA genes in RA aetiology. RMD Open 2019;5:e000893. doi:10.1136/ rmdopen-2019-000893

- Prepublication history and additional material for this paper are available online. To view these files, please visit the journal online (http://dx.doi. org/10.1136/rmdopen-2019000893).

Received 2 January 2019 Revised 12 March 2019 Accepted 27 March 2019
Check for updates

(C) American Society of Regional Anesthesia \& Pain Medicine 2019. Re-use permitted under CC BY-NC. No commercial re-use. Published by BMJ.

${ }^{1}$ Department of Clinical Neuroscience, Karolinska Institutet, Stockholm, Sweden ${ }^{2}$ Instititute of Environmental Medicine, Karolinska Institutet, Stockhom, Sweden ${ }^{3}$ Department of Mathematics, Stockholm university, Stockholm, Sweden ${ }^{4}$ Department of Medicine, Karolinska Institutet, Stockholm, Sweden

Correspondence to Dr Anna Karin Hedström; anna.hedstrom@ki.se

\title{
Interplay between alcohol, smoking and HLA genes in RA aetiology
}

\author{
Anna Karin Hedström, ${ }^{\oplus 1,2}$ Ola Hössjer, ${ }^{3}$ Lars Klareskog, ${ }^{4}$ Lars Alfredsson ${ }^{2}$
}

\section{ABSTRACT}

Objectives The relationship between alcohol consumption and risk for rheumatoid arthritis (RA) is incompletely understood. We aimed to determine the influence of alcohol on anticitrullinated protein antibody (ACPA) positive and ACPA-negative RA and investigate potential interactions between alcohol consumption, smoking and the presence of human leucocyte antigen (HLA)-DRB1shared epitope (SE).

Methods A Swedish population-based case-control study with incident cases of RA was used (3353 cases, 2836 matched controls). Subjects with different HLA-DRB1-SE status, smoking and alcohol consumption were compared regarding risk of ACPA-positive and ACPA-negative RA, by calculating OR with $95 \% \mathrm{Cl}$ employing logistic regression. Interaction on the additive scale between alcohol, HLADRB1-SE and smoking was estimated by calculating the attributable proportion (AP) due to interaction.

Results Compared with non-drinking, low and moderate alcohol consumption was dose dependently associated with a reduced risk of ACPA-positive and ACPA-negative RA. Independent of smoking habits, non-drinking and the presence of HLA-DRB1-SE interacted to increase the risk of ACPA-positive RA. Among HLA-DRB1-SE positive subjects, there was also a significant interaction between non-drinking and smoking with regard to risk for ACPApositive RA. A three-way interaction was observed between alcohol, smoking and HLA-DRB1-SE with regard to risk for ACPA-positive RA (AP 0.7, 95\% $\mathrm{Cl} 0.6$ to 0.8 ) that remained significant when the influence from the two-way interactions was removed (AP $0.4,95 \% \mathrm{Cl} 0.2$ to 0.6 ). Conclusions Our findings emphasize the need to investigate complex interactions between several environmental and genetic factors in order to better understand the etiology of RA. Whereas of great interest in an aetiological perspective, the finding of a protective role of alcohol on risk for RA must, however, be interpreted with caution in a clinical and public health perspective.

\section{INTRODUCTION}

Rheumatoid arthritis (RA) is a chronic inflammatory disease affecting about $1 \%$ of the population. Autoantibodies such as rheumatoid factor (RF) and anticitrullinated protein antibodies (ACPA) are present in the majority of RA cases. ${ }^{1-3}$ ACPA and/or RF positive and negative RA differ in several important

\section{Key messages}

What is already known about this subject?

- Previous studies have been inconclusive regarding the impact of alcohol consumption on the risk of rheumatoid arthritis (RA) and knowledge concerning a potential interplay between alcohol consumption, smoking and the presence of human leucocyte antigen (HLA)-DRB1-shared epitope (SE) in RA aetiology is very limited.

What does this study add?

- Low and moderate alcohol consumption is dose dependently associated with a reduced risk of both anticitrullinated protein antibody (ACPA) positive and ACPA-negative RA.

- Independent of smoking habits, non-drinking and the presence of HLA-DRB1-SE interact to increase the risk for ACPA-positive RA.

How might this impact on clinical practice?

- Our findings emphasise the need to investigate several environmental and genetic factors together in research on aetiology and pathogenesis of RA.

- The mechanisms behind the protective effects of alcohol consumption on risk for RA need to be further investigated.

aspects and have different underlying genetic and environmental risk factors . ${ }^{4-6}$

The relationship between alcohol and RA risk incompletely understood. Alcohol consumption suppresses the synthesis of inflammatory cytokines and chemokines ${ }^{7}$ and has been shown to reduce inflammation and response to antigens in both animals and humans. ${ }^{8-10}$ Several previous studies, but not all, have indicated an inverse relationship between alcohol consumption and risk for RA. ${ }^{11-14}$ There are also inconsistent results concerning the impact of alcohol consumption on risk for the subsets of RA defined by the presence or absence of RF and/or ACPA. ${ }^{11} 1215$ Several systematic reviews and meta-analyses have supported the concept of an inverse relationship between alcohol consumption and $\mathrm{RA},{ }^{16}{ }^{17}$ but knowledge is still lacking concerning the effects of different 
doses of alcohol consumption in RA overall as well as in different subsets of the disease and the potential interplay between alcohol and other risk factors. In order to address some of these remaining questions, we used an updated and enlarged data set from the Swedish population-based case-control study Epidemiologic Investigations of Rheumatoid Arthritis (EIRA) to determine the influence of alcohol on RA risk overall and on disease subsets based on the presence or absence of ACPA. We also set out to investigate potential interactions between alcohol consumption, smoking and the presence of human leucocyte antigen (HLA)-DRB1-shared epitope (SE).

\section{METHODS}

\section{Study design and study subjects}

This report is based on data from EIRA, which is a population-based case-control study comprising the population aged 18-91 years in the middle and southern parts of Sweden. Incident cases of RA were recruited via all hospital-based rheumatology units in the study area during the period between November 1996 and September 2014. Cases were diagnosed by a rheumatologist according to the American College of Rheumatology 1987 criteria. The mean duration between the first notice of any signs of joint inflammation by the patient and the diagnosis of RA by a rheumatologist was 0.9 years (SD 1.2). For cases recruited between November 1996 and October 2005, one control was randomly selected from the national population register, frequency matched by age in 5-year age strata, gender and residential area (EIRA I). For cases recruited between October 2005 and September 2014, two controls were selected using the same matching criteria (EIRA II).

\section{Data collection}

Information regarding environmental and lifestyle factors was collected using a standardised questionnaire filled out at the time of diagnosis. The response proportion was 92\% for the cases and $75 \%$ for the controls. All participants who filled out a questionnaire were asked to donate blood samples and those who did not donate blood were excluded as were those who could not provide detailed information on alcohol consumption or smoking habits. A flow chart presenting the number of cases and controls is presented in the online supplementary table 1 .

\section{Definition of exposures}

Information on alcohol consumption was obtained by asking about current and previous alcohol consumption habits, which allowed quantification of the average alcohol intake in gram per week.

In EIRA1, subjects provided information on alcohol status during the last 12 months (drinking or non-drinking). They reported their current alcohol intake and stated, on a seven-point scale, whether this consumption was higher, equal or lower than their usual alcohol consumption. In EIRA2, subjects were asked whether they were drinkers, past drinkers or never drinkers. Drinkers reported their habitual drinking habits and stated whether their alcohol consumption was higher, equal or lower compared with 5 years ago. Past drinkers were asked at what age they stopped drinking alcohol. All subjects were categorised into the following four groups based on the distribution of current alcohol intake in gram per week among controls; non-drinkers, low consumption (below or equal to the median), moderate consumption (above the median but below or equal to the 75th percentile) and high consumption (above the 75th percentile).

Detailed information on smoking was obtained by asking about current and previous smoking habits. For each case, the year of disease onset was defined as the index year, and the corresponding controls were given the same index year. Subjects who had smoked before or during the index year were defined as ever smokers and those who had never smoked before or at the index year were defined as never smokers.

\section{Biological analyses}

HLA-DRB1 genotypes were obtained as previously described. ${ }^{18}$ The HLA-DRB $1 * 01$, HLA-DRB $1 * 04$ and HLA-DRB $1 * 10$ alleles were classified as the SE alleles.

Cases were categorised into anticyclic citrullinated peptide 2 (CCP2) positive or anti-CCP2 negative based on the result of Immunoscan-RA Mark2 ELISA test (anti-CCP2 test). An antibody level exceeding $25 \mathrm{AU} / \mathrm{mL}$ was regarded as ACPA positive. RF positivity or RF negativity was determined locally, by the unit entering the case into the study.

\section{Statistical analysis}

Using unconditional logistic regression models, ${ }^{19}$ the occurrence of ACPA-positive and ACPA-negative RA in subjects with different alcohol consumption habits was compared with that in non-drinkers by calculating OR with $95 \%$ CI. Women and men were also analysed separately. Trend test for a dose-response relationship regarding alcohol consumption and risk of ACPA-positive and ACPA-negative RA was performed by using a continuous variable for the amount of alcohol consumed per week ( $\mathrm{g}$ ) in a logistic regression model. In order to illustrate the influence of the weekly amount of alcohol consumption (number of glasses of alcohol per week) on

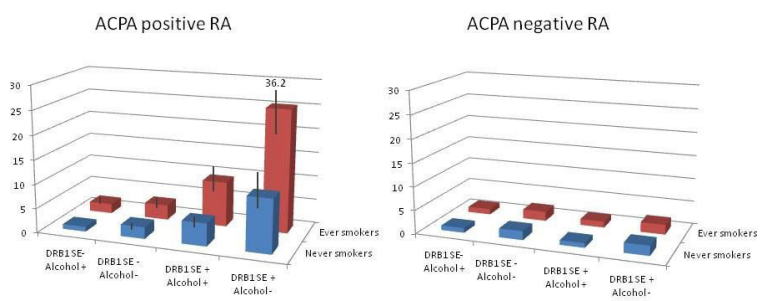

Figure 1 Dose-response relationship between the weekly amount of alcohol consumption and risk of developing RA. ACPA, anticitrullinated protein antibodies; RA, rheumatoid arthritis. 
Table 1 OR with $95 \% \mathrm{Cl}$ of developing RA for different categories of alcohol consumers compared with never drinkers, in total and stratified by gender

\begin{tabular}{|c|c|c|c|c|c|c|}
\hline \multirow[b]{2}{*}{ Alcohol consumption } & \multicolumn{2}{|l|}{ Total } & \multicolumn{2}{|c|}{ ACPA-positive RA } & \multicolumn{2}{|c|}{ ACPA-negative RA } \\
\hline & CA/CO* & OR $(95 \% \mathrm{Cl}) \dagger$ & CA/CO* & OR $(95 \% \mathrm{Cl}) \dagger$ & CA/CO* & OR $(95 \% \mathrm{Cl}) \dagger$ \\
\hline Never & $930 / 431$ & 1.0 (reference) & $621 / 431$ & 1.0 (reference) & $309 / 431$ & 1.0 (reference) \\
\hline Low & $1324 / 1180$ & 0.57 (0.49 to 0.66$)$ & $883 / 1180$ & 0.54 (0.46 to 0.64$)$ & $441 / 1180$ & 0.62 (0.51 to 0.76 \\
\hline Moderate & $624 / 627$ & 0.49 (0.41 to 0.58$)$ & $393 / 627$ & 0.43 (0.36 to 0.52$)$ & $231 / 627$ & 0.59 (0.47 to 0.74 \\
\hline \multirow[t]{2}{*}{ High } & $475 / 598$ & 0.38 (0.31 to 0.45$)$ & $296 / 598$ & 0.33 (0.27 to 0.41$)$ & $179 / 598$ & 0.47 (0.37 to 0.60 \\
\hline & & $<0.0001 \neq$ & & $<0.0001 \ddagger$ & & $0.005 \ddagger$ \\
\hline \multicolumn{7}{|l|}{ Women } \\
\hline Never & $733 / 353$ & 1.0 (reference) & $492 / 353$ & 1.0 (reference) & $241 / 353$ & 1.0 (reference) \\
\hline Low & $1038 / 963$ & 0.57 (0.48 to 0.68$)$ & $699 / 963$ & 0.54 (0.45 to 0.65$)$ & $339 / 963$ & 0.63 (0.51 to 0.79 \\
\hline Moderate & $421 / 461$ & 0.47 (0.39 to 0.57$)$ & $265 / 461$ & 0.41 (0.33 to 0.51$)$ & $156 / 461$ & 0.60 (0.46 to 0.78 \\
\hline \multirow[t]{2}{*}{ High } & $212 / 295$ & 0.37 (0.29 to 0.47$)$ & $131 / 295$ & 0.31 (0.24 to 0.41$)$ & $81 / 295$ & 0.49 (0.36 to 0.68 \\
\hline & & $<0.0001 \ddagger$ & & $<0.0001 \mp$ & & $0.003 \ddagger$ \\
\hline \multicolumn{7}{|l|}{ Men } \\
\hline Never & $197 / 78$ & 1.0 (reference) & $129 / 78$ & 1.0 (reference) & $68 / 78$ & 1.0 (reference) \\
\hline Low & $286 / 217$ & 0.57 (0.41 to 0.80$)$ & $184 / 217$ & 0.55 (0.38 to 0.79 ) & $102 / 217$ & 0.60 (0.39 to 0.91 \\
\hline Moderate & $203 / 166$ & 0.53 (0.38 to 0.76$)$ & $128 / 166$ & 0.51 ( 0.35 to 0.75$)$ & $75 / 116$ & 0.58 (0.37 to 0.90 \\
\hline \multirow[t]{2}{*}{ High } & $263 / 303$ & 0.39 (0.28 to 0.54$)$ & $165 / 303$ & 0.36 (0.25 to 0.52$)$ & $98 / 303$ & 0.43 (0.28 to 0.66 \\
\hline & & $<0.0001 \ddagger$ & & $<0.0001 \ddagger$ & & $0.2 \ddagger$ \\
\hline
\end{tabular}

Low alcohol consumption means less than approximately four glasses of alcohol per week; moderate alcohol consumption means approximately four to seven glasses of alcohol per week; high alcohol consumption means approximatley eight or more glasses of alcohol per week.

*Number of exposed CA and CO.

†Adjusted for age, gender, residential area, ancestry, smoking, human leucocyte antigen-DRB1 shared epitope and study. $\ddagger P$ value for trend,

ACPA, anticitrullinated protein antibodies; CA, cases; CO, controls; RA, rheumatoid arthritis.

RA risk, we used polynomial regression of order four to fit the regression lines to the estimates of ORs. One glass of alcohol was defined as $12 \mathrm{~g}$ of alcohol.

Potential interactions were analysed using departure of additivity of effects as criterion of interaction and were evaluated by calculating AP with 95\% CI. Before calculating measures of interaction on the additive scale, preventive factors should be recoded into risk factors. ${ }^{20}$ Non-drinking was thus considered a risk factor. We investigated the interaction between non-drinking and HLA-DRB1-SE with regard to risk of ACPA-positive and ACPA-negative RA and stratified the analyses by smoking status. The interaction between smoking and HLA-DRB1-SE was also calculated and stratified by alcohol consumption. Similarly, the potential interaction between non-drinking and smoking was calculated and stratified by HLA-DRB1-SE status. Finally, we investigated a potential three-way interaction between alcohol, smoking and HLA-DRB1-SE with regard to ACPA-positive and ACPA-negative RA. ${ }^{21}$

The relationship between alcohol, smoking and HLA-DRB1-SE was further investigated with regard to each RA subgroup based on both RF and ACPA status.

All analyses were adjusted for age in 5-year age strata, gender, residential area (one of 15 counties), ancestry, study (EIRA I and EIRA II) and when appropriate for smoking, alcohol consumption or HLA-DRB1 status. Ancestry was dichotomised into Swedish or non-Swedish. A subject who was born in Sweden, whose parents had not immigrated, was classified as Swedish.

Adjustments were also made for educational level (university degree or not), exposure to passive smoking (yes or no) and body mass index at inclusion in the study (more or less than $25 \mathrm{~kg} / \mathrm{m}^{2}$ ). However, these factors had minor influence on the results and were not kept in the final analyses.

In order to evaluate the presence of bias due to potential reverse causation, we performed sensitivity analyses restricted to participants who reported that they had not changed their alcohol consumption during the last 5 years prior to diagnosis. All analyses were conducted using Statistical Analysis System (SAS) V.9.4.

\section{RESULTS}

The analyses on alcohol consumption and risk of RA included 3353 cases and 2836 matched controls. Characteristics of cases and controls, by alcohol consumption habits, are presented in the online supplementary 


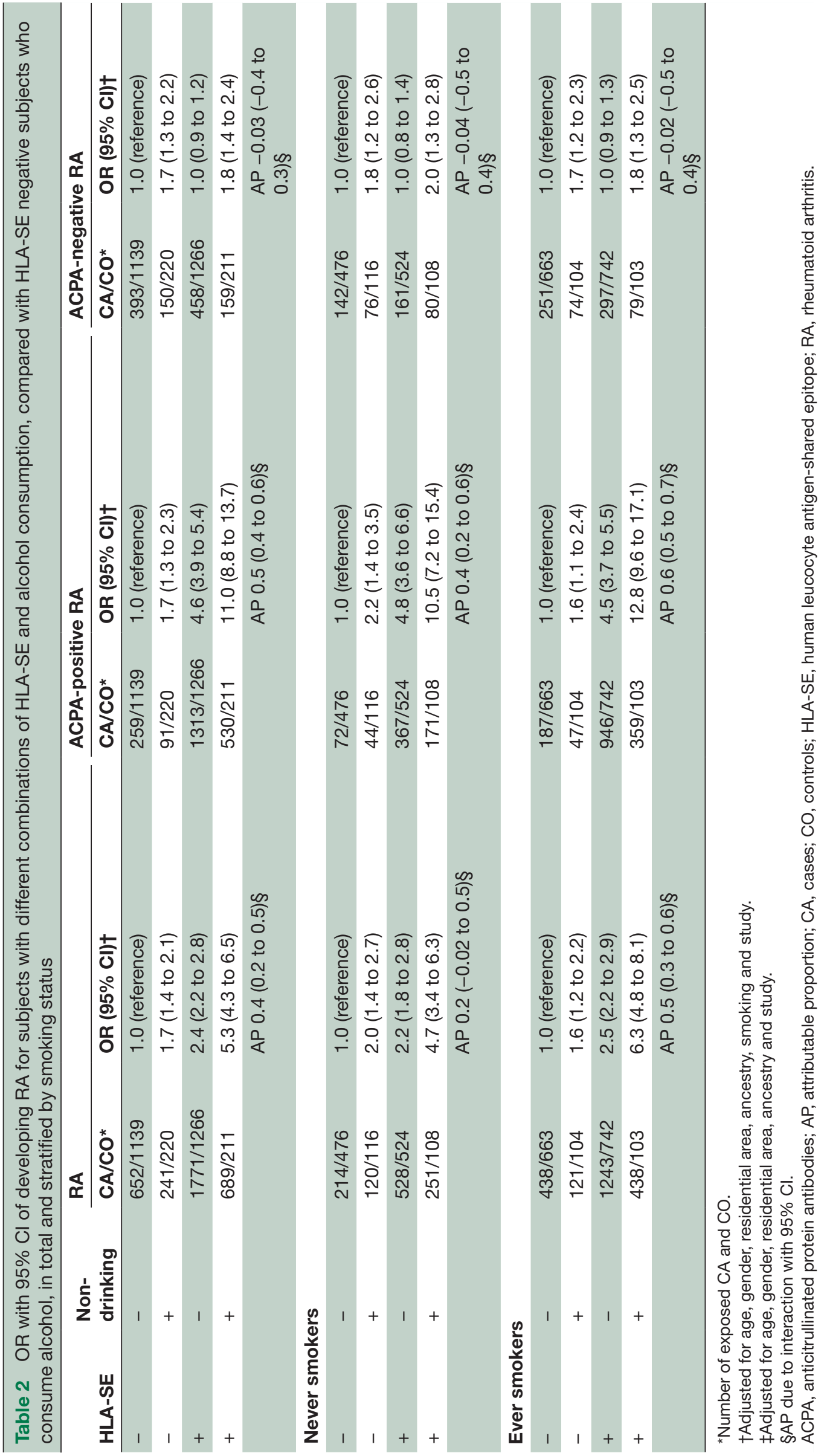


Table 3 OR with $95 \% \mathrm{Cl}$ of developing RA for subjects with different combinations of alcohol and smoking habits, compared with never smokers who consume alcohol, in total and stratified by HLA-SE status

\begin{tabular}{|c|c|c|c|c|c|c|c|}
\hline \multirow{2}{*}{$\begin{array}{l}\text { Non- } \\
\text { drinking }\end{array}$} & \multirow[b]{2}{*}{ Smoking } & \multicolumn{2}{|l|}{ RA } & \multicolumn{2}{|c|}{ ACPA-positive RA } & \multicolumn{2}{|c|}{ ACPA-negative RA } \\
\hline & & $\mathrm{CA} / \mathrm{CO}^{*}$ & OR (95\% Cl)† & $\mathrm{CA} / \mathrm{CO}^{*}$ & OR $(95 \% \mathrm{Cl}) \dagger$ & CA/CO* & OR (95\% Cl)† \\
\hline- & - & $742 / 1000$ & 1.0 (reference) & $439 / 1000$ & 1.0 (reference) & $303 / 1000$ & 1.0 (reference) \\
\hline- & + & $1681 / 1405$ & 1.7 (1.5 to 1.9$)$ & $1133 / 1405$ & 2.0 (1.7 to 2.3 ) & $548 / 1405$ & 1.2 (1.0 to 1.4$)$ \\
\hline+ & - & $371 / 224$ & 2.1 (1.7 to 2.6$)$ & $215 / 224$ & 2.2 (1.7 to 2.8$)$ & $156 / 224$ & 2.0 (1.5 to 2.5$)$ \\
\hline \multirow[t]{2}{*}{+} & + & $559 / 207$ & 3.5 (2.9 to 4.3 ) & $406 / 207$ & 4.8 (3.8 to 5.9 ) & $153 / 207$ & 2.0 (1.5 to 2.6$)$ \\
\hline & & & 0.2 (0.03 to 0.4$)$ & & $0.3(0.2$ to 0.5$)$ & & $-0.08(-0.4$ to 0.3$)$ \\
\hline
\end{tabular}

\section{HLA-SE negative}

\begin{tabular}{|c|c|c|c|c|c|c|c|}
\hline- & - & $214 / 476$ & 1.0 (reference) & $72 / 476$ & 1.0 (reference) & $142 / 476$ & 1.0 (reference) \\
\hline - & + & $438 / 663$ & 1.5 (1.2 to 1.9$)$ & $187 / 663$ & 2.1 (1.5 to 2.8 ) & $251 / 663$ & 1.4 (1.0 to 1.8$)$ \\
\hline+ & - & $120 / 116$ & 2.2 (1.6 to 3.0$)$ & $44 / 116$ & 2.6 (1.6 to 4.1$)$ & $76 / 116$ & 2.3 (1.6 to 3.3$)$ \\
\hline \multirow[t]{2}{*}{+} & + & $121 / 104$ & 2.4 (1.8 to 3.4 ) & $47 / 104$ & 3.2 (2.1 to 5.1$)$ & $74 / 104$ & 2.4 (1.6 to 3.5$)$ \\
\hline & & & $-0.04(-0.4$ to 0.4$)$ & & $-0.09(-0.5$ to 0.4$)$ & & $-0.1(-0.6$ to 0.4$)$ \\
\hline \multicolumn{8}{|c|}{ HLA-SE positive } \\
\hline - & - & $528 / 524$ & 1.0 (reference) & $367 / 524$ & 1.0 (reference) & $161 / 524$ & 1.0 (reference) \\
\hline- & + & $1243 / 742$ & 1.7 (1.5 to 2.0$)$ & $946 / 742$ & $2.0(1.7$ to 2.4$)$ & $297 / 742$ & $1.2(1.0$ to 1.5$)$ \\
\hline+ & - & $251 / 108$ & 2.2 (1.7 to 2.9 ) & $171 / 108$ & $2.3(1.7$ to 3.1$)$ & $80 / 108$ & 1.9 (1.3 to 2.8$)$ \\
\hline \multirow[t]{2}{*}{+} & + & $438 / 103$ & 4.1 (3.2 to 5.4$)$ & $359 / 103$ & 5.3 (4.0 to 7.1$)$ & $79 / 103$ & 1.9 (1.3 to 2.8$)$ \\
\hline & & & 0.3 (0.1 to 0.5$)$ & & 0.4 (0.2 to 0.6$)$ & & $-0.1(-0.6$ to 0.4$)$ \\
\hline
\end{tabular}

${ }^{*}$ Number of exposed CA and CO.

†Adjusted for age, gender, residential area, ancestry, HLA-SE status and study.

$\ddagger$ Adjusted for age, gender, residential area, ancestry and study.

ACPA, anticitrullinated protein antibodies; CA, cases; CO, controls; HLA-SE, human leucocyte antigen-shared epitope; RA, rheumatoid arthritis.

table 2. Compared with non-drinkers, alcohol consumption was associated with a close to $50 \%$ reduced risk of ACPA-positive RA (OR $0.53,95 \%$ CI 0.42 to 0.67 ) and a $30 \%$ reduced risk of ACPA-negative RA (OR 0.65, 95\% CI 0.50 to 0.85 ) with an inverse dose-response relationship up to a level of approximately 12 drinks per week $(\mathrm{p}<0.0001$ in both RA subsets) after which the trend towards protection was interrupted (table 1, figure 1).

With regard to ACPA-positive RA, there was a significant interaction between non-drinking and HLA-DRB1-SE, irrespective of smoking habits (table 2) and between smoking and HLA-DRB1-SE, irrespective of alcohol consumption (online supplementary table 3). There were no interactions with regard to ACPA-negative RA.

Furthermore, among HLA-DRB1-SE positive subjects, there was also a significant interaction between non-drinking and smoking with regard to ACPA-positive RA (table 3). No interaction was observed among HLA-DRB1-SE negative subjects.

A three-way interaction was observed between non-drinking, smoking and HLA-DRB1-SE with regard to ACPA-positive RA (AP 0.7, 95\% CI 0.6 to 0.8) that remained significant when the influence from the two-way interactions was removed (AP $0.4,95 \%$ CI 0.2 to 0.6 ) (table 4). The interaction between HLA-DRB1-SE and non-drinking, among never smokers and ever smokers, is illustrated in figure 2.
Both smoking and non-drinking independently increased the risk of ACPA-negative RA, but there was no interaction between the two risk factors (AP $-0.08,95 \%$ CI -0.4 to -0.3 ) (table 4 ). The results did not change significantly when the cases were further categorised by RF status (online supplementary table 4-6).

The inverse association between alcohol consumption and RA risk remained similar when we restricted the analyses to include subjects who had not changed their alcohol consumption during the last 5 years ( $p$ for trend $0.0002)$. The interaction between non-drinking and DRB1 SE with regard to ACPA-positive RA, and between non-drinking and smoking among DRB1 SE positive subjects, also remained similar.

\section{DISCUSSION}

Our results demonstrate an inverse dose-response relationship between low and moderate alcohol consumption and risk of both ACPA-positive and ACPA-negative $\mathrm{RA}$, with a possible reduction of the protective effect at higher consumption when both subsets of RA were analysed together. With regard to ACPA-positive RA, interactions were observed between HLA-DRB1-SE and alcohol and between HLA-DRB1-SE and smoking. Among HLA-DRB1-SE positive subjects, there was also a significant interaction between non-drinking and smoking with 


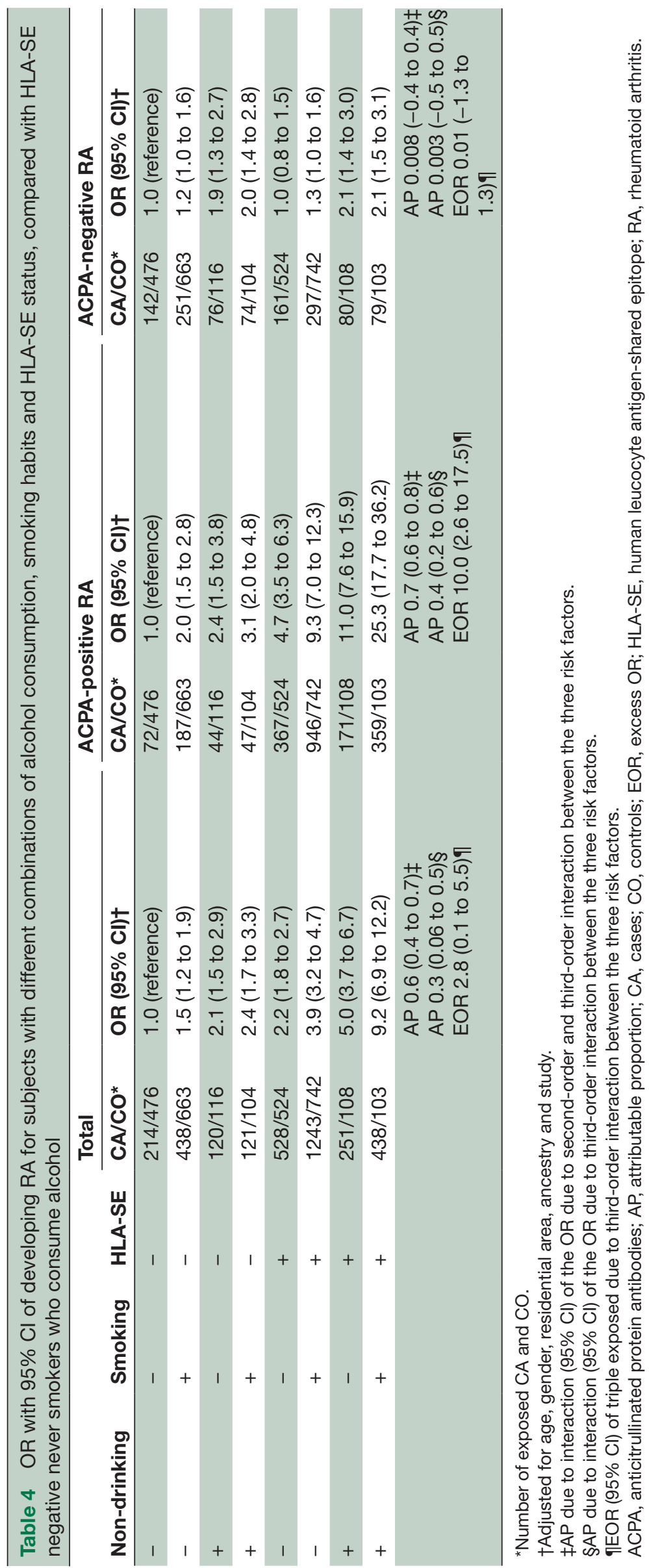




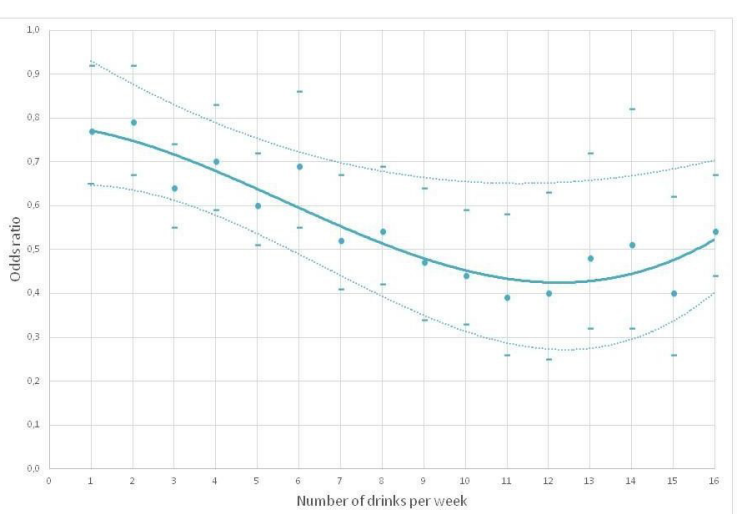

Figure 2 Interaction between HLA-DRB1 shared epitope and non-drinking with regard to risk of developing ACPApositive and ACPA-negative RA, among never smokers and ever smokers. Based on data from table 4. ACPA, anticitrullinated protein antibodies; HLA, human leucocyte antigen; RA, rheumatoid arthritis.

regard to ACPA-positive RA. A significant three-way interaction was observed among the triple exposed regarding risk of developing ACPA-positive RA.

Several autoimmune diseases have been inversely associated with alcohol consumption, such as thyroid diseases, ${ }^{2223}$ systemic lupus erythematosus, ${ }^{24}$ autoimmune diabetes $^{25}$ and multiple sclerosis. ${ }^{26}$ Alcohol has dose-dependent immunomodulatory properties and has been shown to attenuate innate inflammatory responses in humans which is compatible with our finding that alcohol consumption is inversely related both to ACPA-positive and ACPA-negative RA. ${ }^{78}$ In studies on mice, alcohol reduces the incidence and severity of collagen-induced arthritis with a simultaneous downregulation of leucocyte migration and upregulation of testosterone secretion. ${ }^{10}$ Since most environmental factors for RA identified so far have mainly been related to ACPA-positive RA, it is especially interesting that alcohol is inversely related to ACPA-negative RA with about the same magnitude as for ACPA-positive RA.

A similar interaction between non-drinking and smoking has been observed with regard to risk of developing multiple sclerosis. ${ }^{26}$ Alcohol thus seems to have a similar impact on RA and multiple sclerosis, which are both complex Th1 driven inflammatory diseases. However, knowledge concerning the molecular mechanisms underlying the specific interactions presented in this report are very limited.

Our study was designed as a case-control study with incident cases and information regarding environmental exposures and lifestyle factors was collected retrospectively. Recall bias was minimised by using incident cases of RA. We took great effort to obtain information on lifestyle factors and environmental exposures in an identical way for the cases and the controls. Furthermore, the questionnaire contained a wide range of questions regarding many potential environmental risk factors and no section in the questionnaire was given prime focus.
Reverse causation may be a concern since patients with RA may have been less inclined to consume alcohol due to their decreased general well-being before the diagnosis. However, in EIRA2, only 4\% more cases than controls reduced their alcohol consumption in the 5-year period preceding the RA diagnosis, but without abstaining from alcohol. When the analyses were restricted to subjects who reported that they had not changed their alcohol consumption during the 5 years prior to diagnosis, our findings remained similar.

The observed interaction between DRB1-SE and non-drinking with regard to ACPA-positive RA also alleviates some potential biases in the interpretation of the influence from alcohol consumption on RA risk, since the HLA alleles are not likely to determine exposure habits. We thus consider it unlikely that our findings would be affected by recall bias since such a bias would then depend on HLA types.

Our classification of alcohol in categories of low, moderate and high consumption was based on the distribution among all controls. We examined other ways to classify alcohol exposure. A classification based on the distribution among controls by gender or applying the classification that is used by Statistics Sweden ${ }^{27}$ did only marginally change the results.

When analysing the association between alcohol and RA risk, never drinkers of alcohol were used as the reference category in the main analyses. We also performed analyses of the association between alcohol and risk of RA among drinkers (ie, never drinkers were excluded). Also in this group, there was a statistically significant inverse dose-response relationship between alcohol and RA risk ( $\mathrm{p}$ value for trend $<0.0001$ ).

A potential selection bias may arise when recruiting cases and controls. The proportion of respondents with regard to participation in EIRA was $92 \%$ for cases and $75 \%$ for controls. Since the structure of the Swedish public healthcare system provides equal access to medical services for all Swedish citizens, we know that almost all cases of RA are referred to public rheumatology units, ${ }^{28}$ and it is not likely that the few unidentified cases would cause a substantial bias in our calculations. Selection bias among controls is also likely to be modest since controls were selected from the population and the prevalence of smoking and alcohol consumption among controls, seen as an indicator of life style, was in line with that of the general population at equivalent ages. ${ }^{29}$ Furthermore, a previous methodological study from our group has shown that there is no significant differences between those who provided a blood sample and those who did not, ${ }^{28}$ indicating that selection bias did not take place in this step.

In summary, our findings suggest that low and moderate alcohol consumption is inversely associated with both ACPA-positive and ACPA-negative RA. We find the results challenging with regard to how we should relate to and communicate these striking protective effects on RA from a habit with destructive effects for other disease conditions. The findings are also challenging 
with regard to mechanism of action of the protection which are still poorly understood. Better understanding of such mechanisms may help to define ways to achieve protection against RA also by other means than alcohol consumption.

Contributors All authors contributed to the conception or design of the work; the acquisition, analysis or interpretation of data; approved the final version to be published and agreed to be accountable for all aspects of the work in ensuring that questions related to the accuracy or integrity of any part of the work are appropriately investigated and resolved. AKH drafted the work and LA and LK revised it critically for important intellectual content.

Funding The EIRA study was supported by grants from the Swedish Medical Research Council, the Swedish Council for Health, Working life and Welfare, King Gustaf V:s 80-year foundation, the Swedish Rheumatism Foundation, Stockholm County Council, the Swedish Society for Medical Research, the insurance company AFA, and the IMI-supported RTCure project.

Competing interests None declared.

Patient consent for publication Not required.

Ethics approval All aspects of the study were approved by the Regional Ethical Review Board at Karolinska Institutet.

Provenance and peer review Not commissioned; externally peer reviewed.

Data availability statement All data relevant to the study are included in the article or uploaded as supplementary information.

Open access This is an open access article distributed in accordance with the Creative Commons Attribution Non Commercial (CC BY-NC 4.0) license, which permits others to distribute, remix, adapt, build upon this work non-commercially, and license their derivative works on different terms, provided the original work is properly cited, an indication of whether changes were made, and the use is non-commercial. See: http://creativecommons.org/licenses/by-nc/4.0/.

\section{REFERENCES}

1. Schellekens $G A$, Visser $H$, de Jong BA, et al. The diagnostic properties of rheumatoid arthritis antibodies recognizing a cyclic citrullinated peptide. Arthritis Rheum 2000;43:155-63.

2. Avouac J, Gossec L, Dougados M. Diagnostic and predictive value of anti-cyclic citrullinated protein antibodies in rheumatoid arthritis: a systematic literature review. Ann Rheum Dis 2006;65:845-51.

3. Nishimura K, Sugiyama D, Kogata Y, et al. Meta-analysis: diagnostic accuracy of anti-cyclic citrullinated peptide antibody and rheumatoid factor for rheumatoid arthritis. Ann Intern Med 2007;146:797-808.

4. Klareskog L, Stolt P, Lundberg K, et al. A new model for an etiology of rheumatoid arthritis: smoking may trigger HLA-DR (shared epitope)-restricted immune reactions to autoantigens modified by citrullination. Arthritis Rheum 2006;54:38-46.

5. Karlson EW, Chang S-C, Cui J, et al. Gene-environment interaction between HLA-DRB1 shared epitope and heavy cigarette smoking in predicting incident rheumatoid arthritis. Ann Rheum Dis 2010:69:54-60.

6. Hedström AK, Rönnelid J, Klareskog L, et al. Smoking, HLAgenes and serology in rheumatoid arthritis; complex relationships investigated in the Swedish EIRA case-control study. Arthritis Rheumatol 2019.

7. Mandrekar P, Catalano D, White B, et al. Moderate alcohol intake in humans attenuates monocyte inflammatory responses: inhibition of nuclear regulatory factor kappa B and induction of interleukin 10. Alcoholism Clin Exp Res 2006;30:135-9.
8. Waldschmidt TJ, Cook RT, Kovacs EJ. Alcohol and inflammation and immune responses: summary of the 2005 alcohol and immunology research Interest Group (AIRIG) meeting. Alcohol 2006;38:121-5.

9. Boé DM, Nelson S, Zhang P, et al. Alcohol-induced suppression of lung chemokine production and the host defense response to Streptococcus pneumoniae. Alcohol Clin Exp Res 2003;27:1838-45.

10. Jonsson I-M, Verdrengh M, Brisslert M, et al. Ethanol prevents development of destructive arthritis. Proceedings of the National Academy of Sciences 2007;104:258-63.

11. Källberg $\mathrm{H}$, Jacobsen $\mathrm{S}$, Bengtsson $\mathrm{C}$, et al. Alcohol consumption is associated with decreased risk of rheumatoid arthritis: results from two Scandinavian case-control studies. Ann Rheum Dis 2009;68:222-7.

12. Maxwell JR, Gowers IR, Moore DJ, et al. Alcohol consumption is inversely associated with risk and severity of rheumatoid arthritis. Rheumatology 2010;49:2140-6.

13. Lu B, Solomon $\mathrm{DH}$, Costenbader $\mathrm{KH}$, et al. Alcohol consumption and risk of incident rheumatoid arthritis in women: a prospective study. Arthritis Rheumatol 2014;66:1998-2005.

14. Rodríguez LAG, Tolosa LB, Ruigómez A, et al. Rheumatoid arthritis in UK primary care: incidence and prior morbidity. Scand J Rheumatol 2009;38:173-7.

15. Heliövaara M, Aho K, Knekt $\mathrm{P}$, et al. Coffee consumption, rheumatoid factor, and the risk of rheumatoid arthritis. Ann Rheum Dis 2000;59:631-5.

16. Scott IC, Tan R, Stahl D, et al. The protective effect of alcohol on developing rheumatoid arthritis: a systematic review and metaanalysis. Rheumatology 2013;52:856-67.

17. Jin Z, Xiang C, Cai Q, et al. Alcohol consumption as a preventive factor for developing rheumatoid arthritis: a dose-response metaanalysis of prospective studies. Ann Rheum Dis 2014;73:1962-7.

18. Padyukov L, Silva C, Stolt $P$, et al. A gene-environment interaction between smoking and shared epitope genes in HLA-DR provides a high risk of seropositive rheumatoid arthritis. Arthritis Rheum 2004;50:3085-92.

19. Pearce N. Analysis of matched case-control studies. BMJ 2016;25:i969-69.

20. Knol MJ, VanderWeele TJ, Groenwold RHH, et al. Estimating measures of interaction on an additive scale for preventive exposures. Eur J Epidemiol 2011;26:433-8.

21. Hedström AK, Hössjer O, Katsoulis M, et al. Organic solvents and MS susceptibility: interaction with MS risk HLA genes. Neurology 2018;91:e455-62.

22. Carlé A, Pedersen IB, Knudsen N, et al. Moderate alcohol consumption may protect against overt autoimmune hypothyroidism: a population-based case-control study. Eur $J$ Endocrinol 2012;167:483-90.

23. Carlé A, Bülow Pedersen I, Knudsen N, et al. Graves' hyperthyroidism and moderate alcohol consumption: evidence for disease prevention. Clin Endocrinol 2013;79:111-9.

24. Hardy CJ, Palmer BP, Muir KR, et al. Smoking history, alcohol consumption, and systemic lupus erythematosus: a case-contro study. Ann Rheum Dis 1998;57:451-5.

25. Rasouli B, Ahlbom A, Andersson T, et al. Alcohol consumption is associated with reduced risk of type 2 diabetes and autoimmune diabetes in adults: results from the Nord-Trøndelag health study. Diabet Med 2013;30:56-64.

26. Hedström AK, Hillert J, Olsson $\mathrm{T}$, et al. Alcohol as A modifiable lifestyle factor affecting multiple sclerosis risk. JAMA Neurol 2014;71:300-5.

27. Internet-based information. Available: https://www.scb.se/statistik/ publikationer/LE0101_2004I05_BR_LE114SA0701.pdf

28. Bengtsson C, Berglund A, Serra M-L, et al. Non-participation in EIRA: a population-based case-control study of rheumatoid arthritis. Scand J Rheumatol 2010;39:344-6.

29. Internet-based information. Available: http://www.scb.se [Accessed 30 Jun 2018]. 